\title{
Characterization of a polyethylene - polyamide multilayer film using nanoscale infrared spectroscopy and imaging
}

\author{
Mauritz Kelchtermans ${ }^{\mathrm{a}}$, Michael Lo ${ }^{\mathrm{b}}$, Eoghan Dillon ${ }^{\mathrm{b}}$, Kevin Kjoller $^{\mathrm{b}}$, Curtis \\ $\operatorname{Marcott}{ }^{\mathrm{c}, *}$ \\ ${ }^{a}$ ExxonMobil Chemical Europe Inc., Hermeslaan 2, B-1831 Machelen, Belgium \\ b Anasys Instruments, Inc., 325 Chapala Street, Santa Barbara, CA 93101, USA \\ ${ }^{\mathrm{c}}$ Light Light Solutions, P. O. Box 81486, Athens, GA 30608-1484, USA \\ * Corresponding author: Curtis Marcott, Light Light Solutions, P. O. Box 814786, \\ Athens, GA 30608-1484, USA E-mail: marcott@ lightlightsolutions.com;
}

\begin{abstract}
Atomic force microscopy (AFM) and infrared (IR) spectroscopy have been combined in a single instrument (AFM-IR) capable of producing IR spectra and absorption images at sub-micrometer spatial resolution. This new device enables cross sections of multilayer films to be spectroscopically characterized at levels not previously possible. In particular, it was possible to observe nanoscale IR spectroscopic differences, as well as thermal and mechanical property differences, in the tie layers located between the individual polyethylene and polyamide layers of a multilayer cling film of initially unknown structure. It also appears that a two- $\mu$ m-thick barrier layer between two polyamide layers near the center of the multilayer cling film consists of an ethylene-vinyl alcohol copolymer. Mechanical stiffness and thermal property differences are also observed between the various layers in the film. This powerful capability should prove generally useful for reverse engineering complex unknown multilayer film materials, as
\end{abstract}


well as in aiding the intelligent design and coextrusion of superior multilayer film materials.

Keywords: infrared; atomic force microscopy; multilayer films; tie layers; polyethylene; polyamide

\section{Introduction}

Multilayer films are of great importance in many products, especially packaging materials. As multilayer films become thinner and the number of individual layers increase, there is an ever increasing need to characterize these structures. Chemical treatment of the polymer film surfaces and/or the addition of tie layers, can improve the adhesion of the main polymer film layers, which are often incompatible, i.e., not naturally adhering to each other. Without proper binding between chemically different polymer film materials, the layers may eventually pull apart, resulting in failure of the packaging film. Techniques that enable the exploration of the thermal, mechanical, and chemical nature of multilayer films at the important boundary region between layers will aid not only in the development of better packaging materials, but also to reverse engineer all kinds of already manufactured multilayer films.

Fourier transform infrared (FT-IR) attenuated total reflection (ATR) [1-2] spectroscopy is a technique which has been used by many researchers to chemically characterize the surface of polymer film samples. It can be useful for identifying tie layers and surface treatments of the internal layers of multilayer films, assuming they can be delaminated cleanly to expose the internal layers, and assuming those layers are thick 
enough to distinguish them from the subsurface layers. FT-IR microspectroscopy is also widely used for characterizing cross sections of multilayer films. It provides information about the chemical nature of the individual polymers layers, as long as they are at least a few micrometers thick. The spatial resolution limit of conventional transmission FT-IR microspectroscopy is limited by fundamental diffraction limitations associated with the wavelength of light used to make measurements in the mid-IR spectral range (2.5-14 $\mu \mathrm{m})$. Studies using ATR objectives coupled with FT-IR microscopes equipped with focal-plane array (FPA) mercury-cadmium-telluride (MCT) detectors have been used to characterize layers as thin as a few microns when a high refractive index internal reflection element (IRE) like Ge is used as the ATR objective, but it is yet not possible to reach sub-micrometer spatial resolution in FT-IR microscopy, even when using synchrotron radiation as the light source [1-2].

The spatial resolution of Raman spectroscopy depends on the laser excitation wavelength, but is generally higher than FT-IR microspectroscopy. However, Raman spectroscopy suffers from the lack of spectral sensitivity for polar substrates which are often used in multilayer films.

The combination of an atomic force microscope (AFM) with a tunable IR laser source in a single instrument has been shown to be capable of collecting sub-diffraction-limited measurements of IR absorption spectra on sample regions as small as $20 \mathrm{~nm}$ x $20 \mathrm{~nm}$. This technique, known as AFM-IR, is based on the photothermal induced resonance effect (PTIR) [3-8].

The AFM-IR technique used in this work focuses radiation from a tunable IR laser source onto a location on the sample top surface from above [3-8]. Unlike conventional 
FT-IR spectrometry, where a high-sensitivity MCT single-element or FPA detector senses the internally reflected IR beam, the AFM-IR approach uses a sharp AFM tip attached to a cantilever to detect the rapid thermal expansion of the sample caused by absorption of short (10 ns) pulses of IR radiation at a given wavenumber. When the monochromatic laser radiation occurs at a wavenumber that excites molecular vibrations in the sample, the light is absorbed and causes a rapid thermal expansion, which causes the AFM tip and the cantilever to jump and ring down at its natural vibrational resonant frequencies. These motions are sensed by reflecting a second laser beam off the top of the cantilever and detecting the signal with a position-sensitive photodetector. The induced resonance amplitude in the cantilever is proportional to the amount of IR radiation absorbed by the sample [3-8]. The resulting spectrum is obtained by measuring the ringdown amplitudes while tuning the IR source over a range of wavenumbers. The source can cover a broad range of the mid-IR spectrum, allowing continuous measurements to be made over the range from $900 \mathrm{~cm}^{-1}$ to $3600 \mathrm{~cm}^{-1}$. More details regarding the AFM-IR experiment can be found elsewhere [3-8]. AFM-IR spectra are virtually identical to those obtained with conventional transmission FT-IR instruments, which means they can be digitally searched against readily available IR spectral databases.

\section{Experimental}

A small wedge of the cling film was embedded in Devcon Two-ton epoxy (Danvers, MA), and was left to cure overnight at ambient temperature. Excess epoxy was removed to expose the cross section of the film and the surface was roughly polished before being 
sectioned at $-70{ }^{\circ} \mathrm{C}$ in a cryo-ultramicrotome (Microstar Technologies, Huntsville, TX). For atomic force microscope-based infrared spectroscopy (AFM-IR) experiments, 200 nm-thick sections were collected onto a $\mathrm{ZnS}$ window at $-70{ }^{\circ} \mathrm{C}$ and were subsequently returned to room temperature for analysis. Film cross sections delaminated from the embedding epoxy were selected for analysis to minimize infrared signatures from the latter. For nanothermal analysis (nanoTA) and Lorentz contact resonance (LCR) measurements, the remaining stub after sectioning was used for all measurements at ambient temperature.

The $\mathrm{ZnS}$ window with the cling film sample was mounted in the nanoIR2 ${ }^{\mathrm{TM}}$ instrument (Anasys Instruments, Inc., Santa Barbara, CA, USA) for analysis. All AFM topographic images were obtained either in contact mode using a Contact Mode AFM Probe for nanoIR2 (Anasys Instruments, Santa Barbara, CA) or in tapping mode using a Tapping Mode Probe for nanoIR2 (Anasys Instruments, Santa Barbara, CA). Analysis Studio software (version 3.10, Anasys Instruments, Inc., Santa Barbara, CA, USA) was used for data collection and analysis. For the collection of local spectra, a cantilever frequency of $730 \mathrm{kHz}$ was used with a width of $100 \mathrm{kHz}$. The IR laser produced $10 \mathrm{~ns}$ pulses at a repetition rate of $1 \mathrm{kHz}$. The power levels of the incident IR radiation on the sample surface were set to $c a .50 \mu \mathrm{W}$ and the focused laser spot size was about 50 - 60 $\mu \mathrm{m}$. Local spectra were collected over two spectral ranges: $3600-2800 \mathrm{~cm}^{-1}$ and $1800-$ $1200 \mathrm{~cm}^{-1}$, using a data point spacing of $4 \mathrm{~cm}^{-1}$. Ringdowns from 256 laser pulses were co-averaged per data point in a given spectrum. Spectral resolution was determined by the laser line width and estimated to be about $4 \mathrm{~cm}^{-1}$. Spatial resolution of the IR spectra collected using AFM-IR is limited by mechanical and thermal properties of the sample, 
deteriorating for thicker samples. For the $\sim 200 \mathrm{~nm}$-thick cross-sections used in this study, we estimate that spatial resolution of the IR spectra is approximately $50-100 \mathrm{~nm}$.

IR laser power was optimized such that softening due to IR heating was minimized for the spectra shown in the Figures. All spectra were smoothed using a Savitzky-Golay filter that applies a $7^{\text {th }}$ order fit over five points and normalized using Anasys Instrument's Analysis Studio software.

Nanoscale thermal (nanoTA) and nanoscale mechanical (LCR) measurements were made using an afm+TM instrument (Anasys Instruments, Inc., Santa Barbara, CA, USA). A 300- $\mu \mathrm{m}$-long ThermaLever ${ }^{\mathrm{TM}}$ was used for all measurements. For all nanoTA experiments, the heating voltage applied across the ThermaLever was monitored and controlled by the resistance of the AFM tip against its deflection. Local heating of the sample will initially lead to expansion of the sample causing the deflection value of the tip to rise. Heating will halt when sufficient softening leads to puncture of the sample surface by the AFM probe, in which case the deflection value will drop. For the LCR experiments, an AC current is passed along the same ThermaLever. Frequency spectra were swept from $10 \mathrm{kHz}$ to $1 \mathrm{MHz}$ to locate the contact resonance frequencies of the probe in contact with the material. Drive strength, i.e., the amplitude of the AC current, was controlled to increase signal-to-noise ratio without melting the sample. For LCR imaging, the modulation frequency of the AFM cantilever and tip is set to $125 \mathrm{kHz}$, which coincides with the ThermaLever's contact resonance.

\section{Results and discussion}


Figure 1 shows a tapping mode AFM height image and AFM-IR spectra from seven layers of a cling film mainly consisting of PE and PA. The colored marker locations on the AFM image are locations where the corresponding AFM-IR spectra of the same color were obtained. IR spectra collected from layers labeled A and F are consistent with polyethylene. Spectra collected from layers C, E, and G are consistent with a polyamide. The IR spectrum recorded from layer D, which can be considered as the barrier layer in the film, is consistent with polyethylene-co-(vinyl alcohol) (EVOH). In this way the main layers of the material are clearly generically identified. For detailed identification of these materials (for instance the type of branching in the PE, the amount of ethylene in the EVOH), the region between 900 and $600 \mathrm{~cm}^{-1}$ would be very useful. However this spectral range is not accessible with the current instrumentation.

Figure 2 shows a contact mode AFM height image and series of nanoscale thermal analysis curves recorded on the seven-layer cling film cross section. The colored marker locations on the AFM image (spaced $1 \mu \mathrm{m}$ apart) represent locations where the nanothermal measurements were recorded. The red marker locations/thermal curves with lower transition temperatures correspond to the PE layers, with slightly different types of $\mathrm{PE}$ at both sides, as evidenced by the small but distinct differences in their transition temperatures, while the blue markers locations/thermal curves correspond to the PA layers. The green marker locations/thermal curves recorded from the apparent barrier layer between the two PA layers are clearly different, adding further confirmation for the presence of the EVOH layer, with a typical DSC melting point between 158 and $185^{\circ} \mathrm{C}$, depending on the ethylene content in the copolymer. 
Figure 3 shows a contact mode AFM height image and Lorentz Contact Resonance (LCR) mechanical spectra of the same seven-layer cling film mainly consisting of PE and PA layers. The colored marker locations on the AFM image (spaced $1.2 \mu \mathrm{m}$ apart) represent locations where the nanoscale mechanical spectral measurements were recorded. The spectra indicate at least three different mechanical stiffness spectral signatures for the PE, PA, and the EVOH barrier layers, which were previously identified via their AFM-IR spectra.

Figure 4 shows a contact mode AFM height image (top) and a LCR amplitude image (bottom) recorded with the tip modulation frequency fixed at $125 \mathrm{kHz}$, which matches peak mechanical frequency of the polyamide component of the sample. Stiffer PA portions of the film cross section show up lighter (i.e., more intense) than the softer PE layers of the film. The EVOH layer also shows up light because it has a similar peak mechanical frequency to PA. The circled area represents a tie layer between the PE and PA layer which has a slightly different mechanical response. Figure 5 shows a contact mode AFM height image and nanoscale thermal analysis traces of the boundary between a PE and a PA layer in the cling film cross section. The colored marker locations on the AFM image (spaced $100 \mathrm{~nm}$ apart) represent locations where the nanothermal measurements were recorded. The red marker locations correspond to the tie layer between the PE and PA layer, which has a distinctly higher melt temperature than the bulk of the PE.

Figure 6 shows a contact mode AFM height image and AFM-IR spectra from the boundary region between the PE and PA layer of the cling film cross section. The 
colored marker locations on the AFM image are locations where the corresponding AFMIR spectra of the same color were obtained (100 $\mathrm{nm}$ spacing). The spectra recorded within $200 \mathrm{~nm}$ of the boundary have significantly sharper $\mathrm{CH}_{2}$-stretching bands than the areas of the PE further away from the boundary. The center-of-mass peak wavenumber of the $\mathrm{CH}_{2}$ antisymmetric stretching band also shifts to lower wavenumber $\left(2916 \mathrm{~cm}^{-1}\right)$ at the boundary, suggesting more ordered hydrocarbon chains. The plots shown on the right hand side of Figure 6 show the full width at half height (FMHH) and peak wavenumber changes in this band near the boundary. This behavior is consistent with the nanoscale thermal analysis results shown in Fig. 5, and suggests a less branched PE-based tie layer was used to adhere the PE to the PA layer. This PE has very probably been grafted with maleic anhydride to ensure efficient reaction between the anhydride and the amide groups to tie the layers together. Further confirmation of this can be obtained by detailed analysis of the region between 1700 and $1850 \mathrm{~cm}^{-1}$. Clearly, using the combined techniques of nanoscale thermal, nanoscale mechanical and AFM-IR spectroscopic characterization we now can describe the very thin tie-layers in a multilayer film.

Figure 7 shows a contact mode AFM height image and AFM-IR spectra from the boundary region between the PA and the EVOH barrier layer of the cling film cross section. The colored marker locations on the AFM image are locations where the corresponding AFM-IR spectra of the same color were obtained (50 nm spacing). A definite broadening of the NH-stretching band centered at $3296 \mathrm{~cm}^{-1}$ is observed, consistent with a gradual transition between the two layers, from $\mathrm{NH}$ to $\mathrm{OH}$. Here there 
is no indication for a separate tie-layer, which can be explained by the fact that both the PA and EVOH are polar materials, hence not requiring further tie-agents.

\section{Conclusion}

Novel instrumentation combining atomic force microscopy and infrared spectroscopy (AFM-IR) dramatically enhances the ability to analyze thin layers in a multilayer cling film. Spatially resolved infrared spectra provide high confidence in the assignment of the components in the laminate. Even information on thin tie layers, less than two micrometers thick, is readily obtained. Nanothermal analysis and Lorentz Contact Resonance mechanical spectroscopy and imaging provide added dimensions to the thermal and mechanical property distributions over the cross section of the film. These capabilities as a whole provide very valuable information for product development as well as for reverse engineering purposes.

\section{Acknowledgements}

We thank NIST-ATP Award \#70NANB7H7025 and the National Science Foundation award NSF-SBIR 0750512 for providing the financial support. 


\section{References}

[1] H.J. Gulley-Stahl, S.B. Bledsoe, A.P. Evan, A.J. Sommer, Appl. Spectrosc. 64 (2010) $15-22$.

[2] M.J. Nasse, M.J. Walsh, E.C. Mattson, R. Reininger, A. Kajdacsy-Balla, V. Macius, R. Bhargava, C.J. Hirschmugl, Nat. Methods 8 (2011) 413-416.

[3] A. Dazzi, F. Glotin, R. Carminati, J. Appl. Phys. 107 (2010) 124519.

[4] A. Dazzi, R. Prazeres, F. Glotin, J.M. Ortega, M. Al-Sawaftah, M. de Frutos, Ultramicroscopy 108 (2008) 635-641.

[5] B. Lahiri, G.Holland, A. Centrone, Small 9 (2012) 439-445.

[6] C. Prater, K. Kjoller, D. Cook, R. Shetty, G. Meyers, C. Reinhardt, J. Felts, W. King, K. Vodopyanov, A. Dazzi., Microscopy and Analysis 24 (2010) 5-8.

[7] A. Dazzi, C.B. Prater, Q. Hu, D. B. Chase, J. F. Rabolt, C. Marcott, Appl. Spectrosc. 66 (2012) 1365-1384.

[8] C. Marcott, M. Lo, K. Kjoller, C. Prater, I. Noda, Appl. Spectrosc. 65 (2011) 11451150. 


\section{FIGURE CAPTIONS}

Figure 1. Tapping mode AFM height image and AFM-IR spectra from seven layers of a multilayer cling film mainly consisting of polyethylene (PE) and polyamide (PA). The colored marker locations on the AFM image are locations where the corresponding AFMIR spectra of the same color were obtained.

Figure 2. Contact mode AFM height image and nanoscale thermal analysis of a sevenlayer cling film sample mainly consisting of PE and PA. The colored marker locations on the AFM image (spaced $1 \mu \mathrm{m}$ apart) represent locations where the nanothermal measurements were recorded. The red marker locations with lower transition temperature correspond to the PE layers.

Figure 3. Contact mode AFM height image and Lorentz contact resonance (LCR) mechanical spectra of the same seven-layer cling film sample mainly consisting of PE and PA layers. The colored marker locations on the AFM image (spaced $1.2 \mu \mathrm{m}$ apart) represent locations where the nanoscale mechanical spectral measurements were recorded.

Figure 4. Contact mode AFM height image and LCR amplitude image recorded with the tip modulation frequency fixed at $125 \mathrm{kHz}$, which is a prominent peak mechanical frequency of the PA component of the sample. Stiffer PA portions of the film cross section show up lighter (i.e., more intense) than the softer PE layers of the film. The 
circled area represents a tie layer between a PE and PA layer which has a slight, but significantly different mechanical response.

Figure 5. Contact mode AFM height image and nanoscale thermal analysis traces of the boundary between a PE and a PA layer. The colored marker locations on the AFM image (spaced $100 \mathrm{~nm}$ apart) represent locations where the nanothermal measurements were recorded. The red marker locations correspond to the tie layer between the PE and PA layer.

Figure 6. Contact mode AFM height image and AFM-IR spectra from the boundary region between a PE and a PA layer. The colored marker locations on the AFM image are locations where the corresponding AFM-IR spectra of the same color were obtained (100 nm spacing). The plot on the upper right shows the full width at half height of the $\mathrm{CH}_{2}$ antisymmetric stretching peak at $2916 \mathrm{~cm}^{-1}$. The plot on the lower right shows the center-of-mass peak wavenumber of this same absorption band.

Figure 7. Contact mode AFM height image and AFM-IR spectra from the boundary region between a PA and the EVOH barrier film layer of the laminate. The colored marker locations on the AFM image are locations where the corresponding AFM-IR spectra of the same color were obtained (50 $\mathrm{nm}$ spacing). 


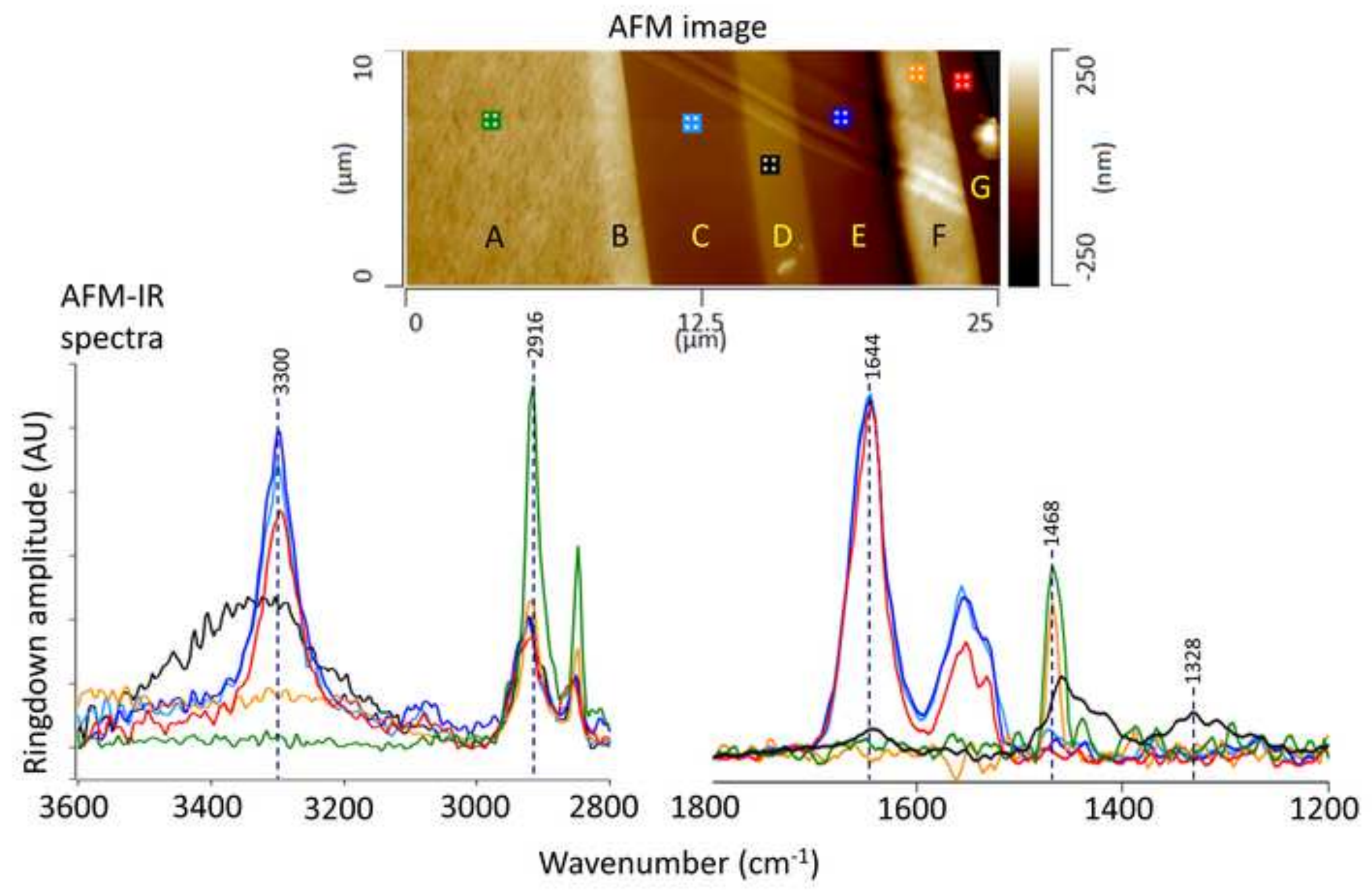




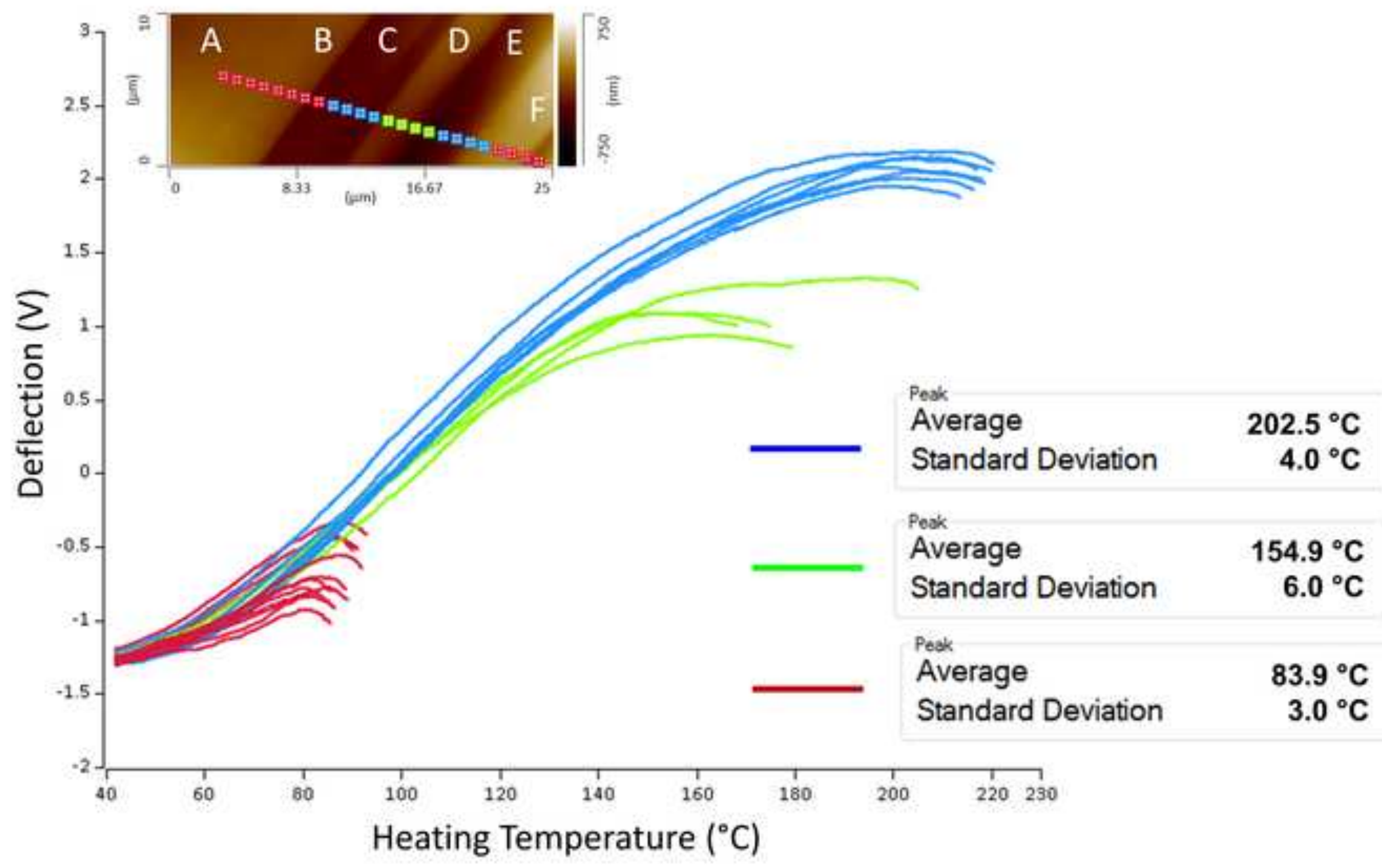




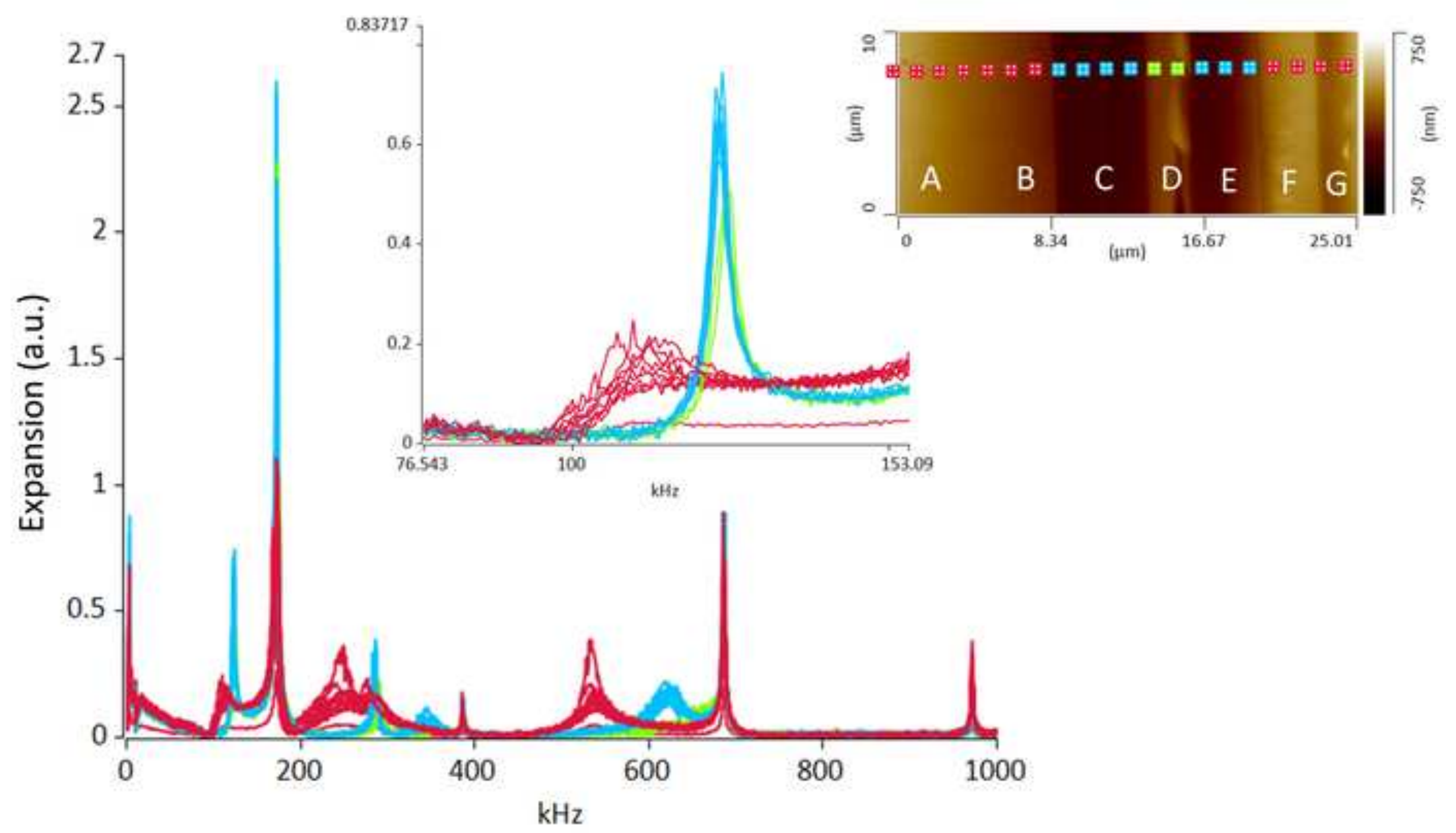




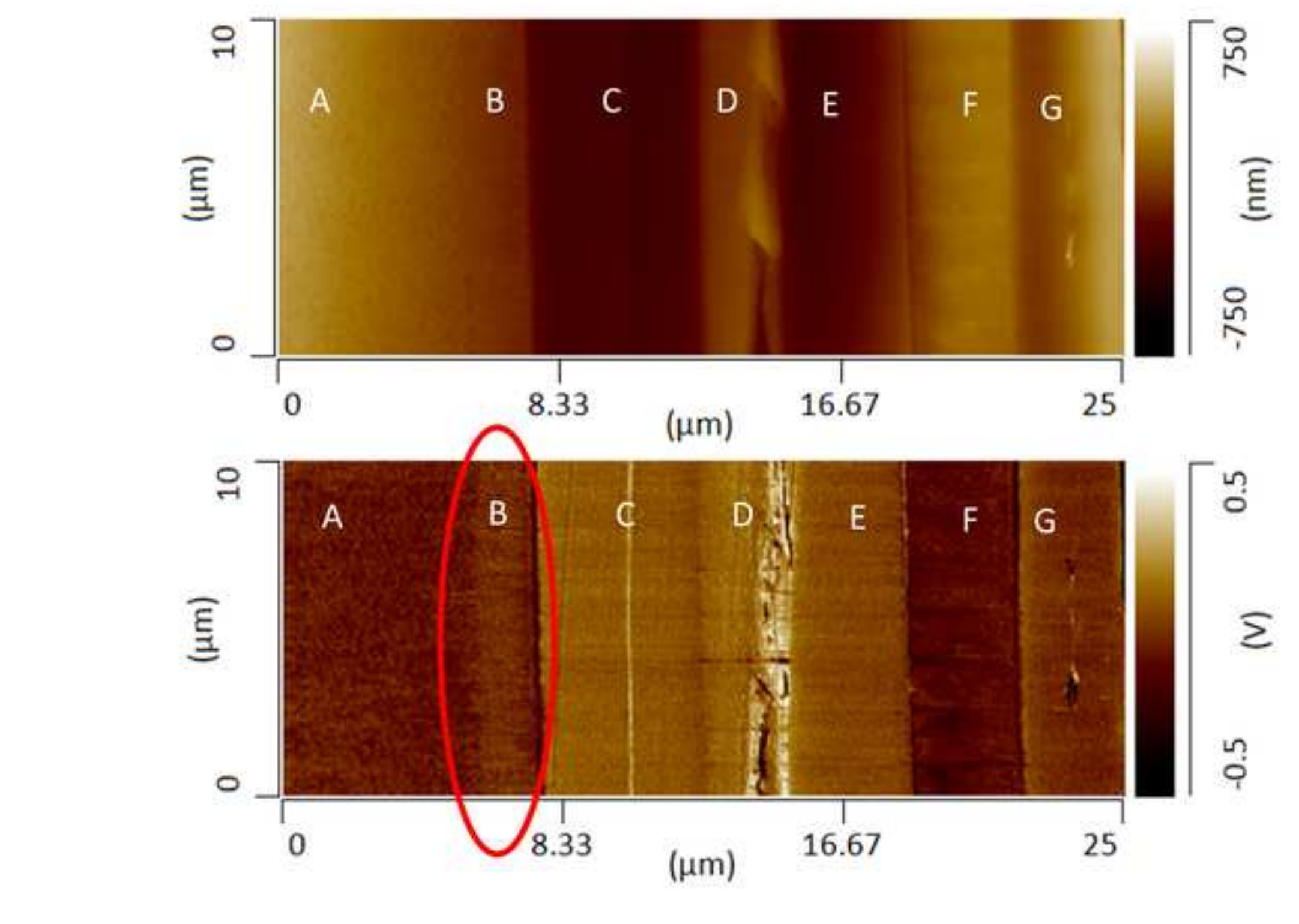

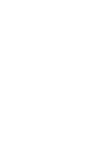




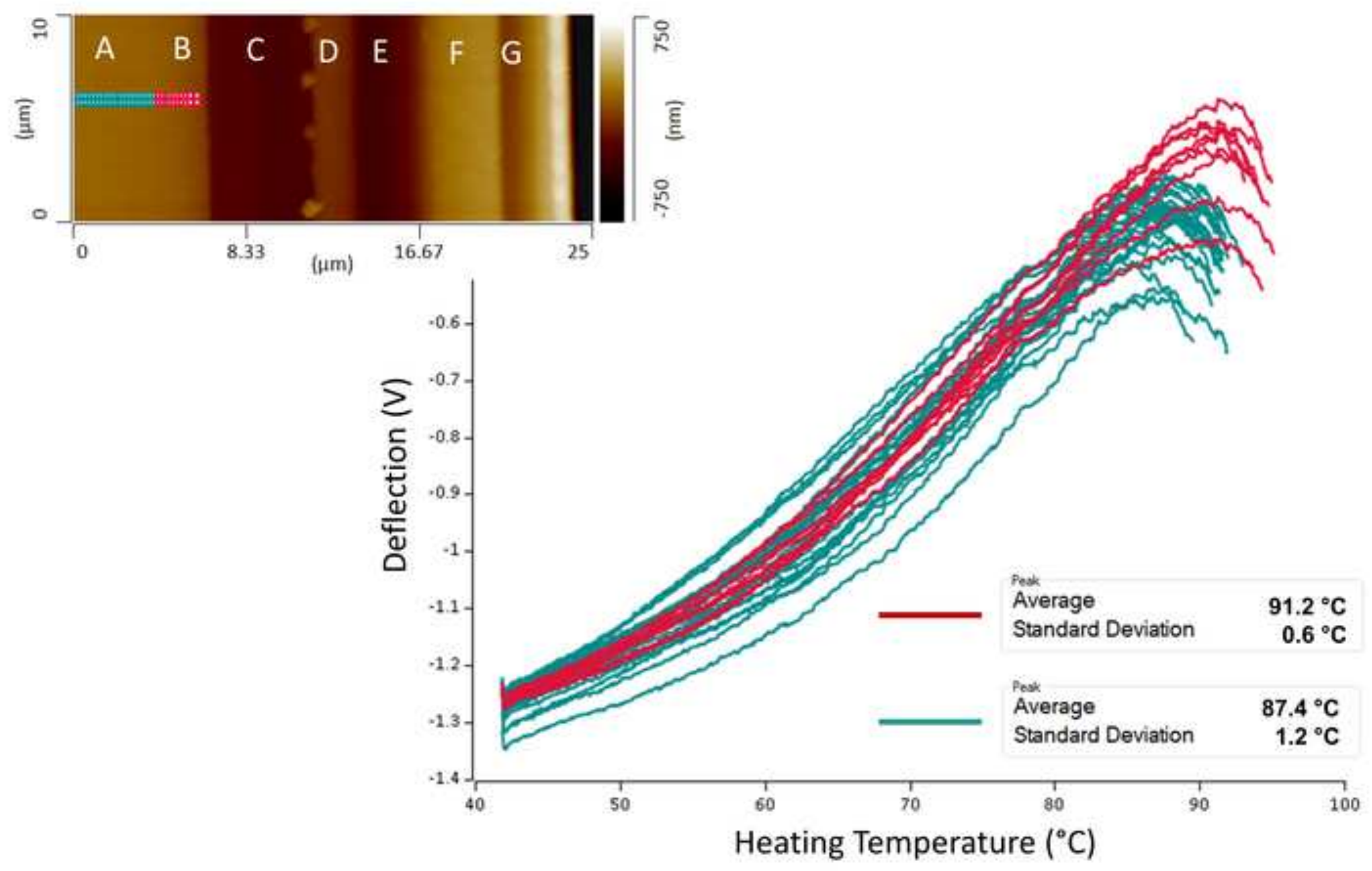



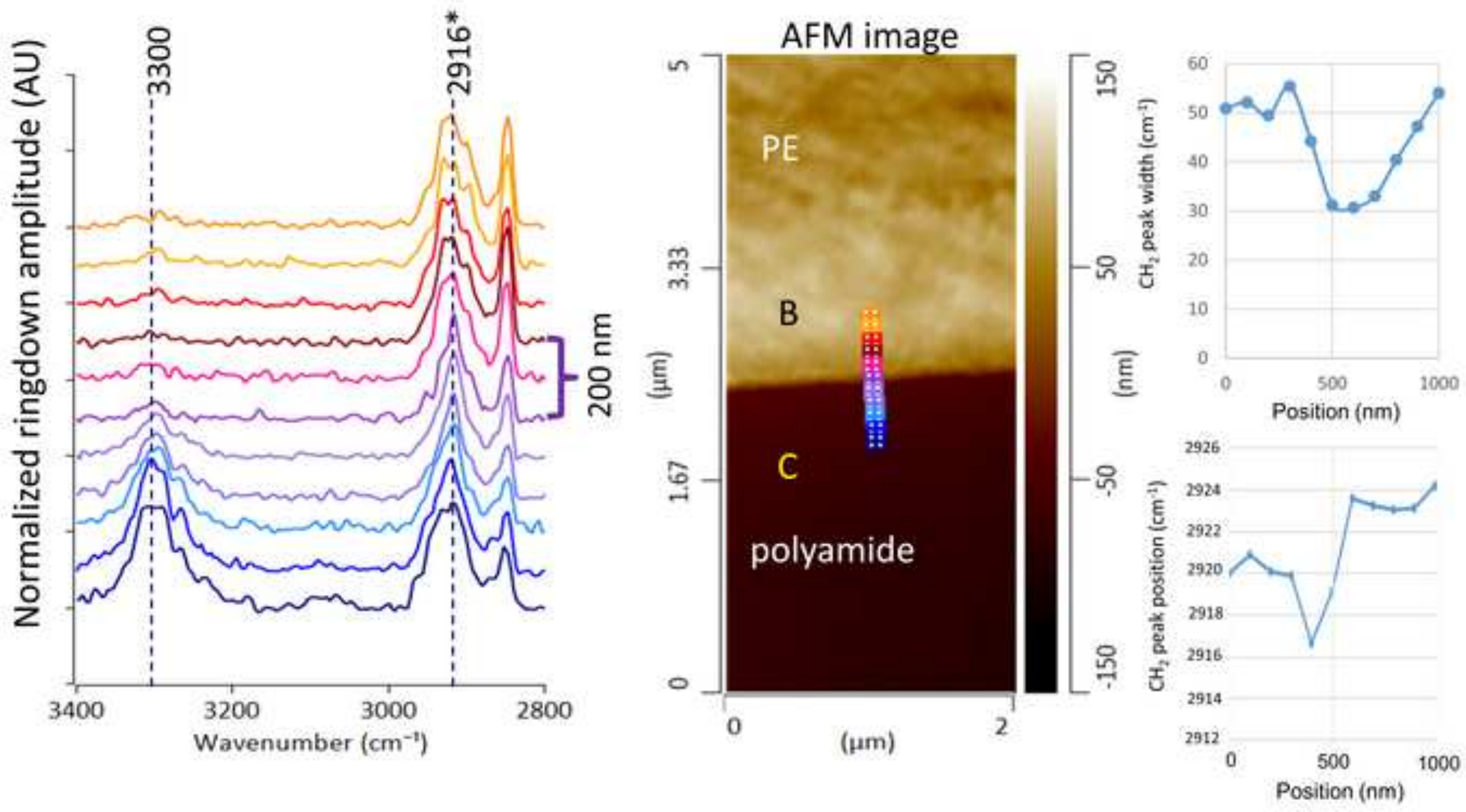


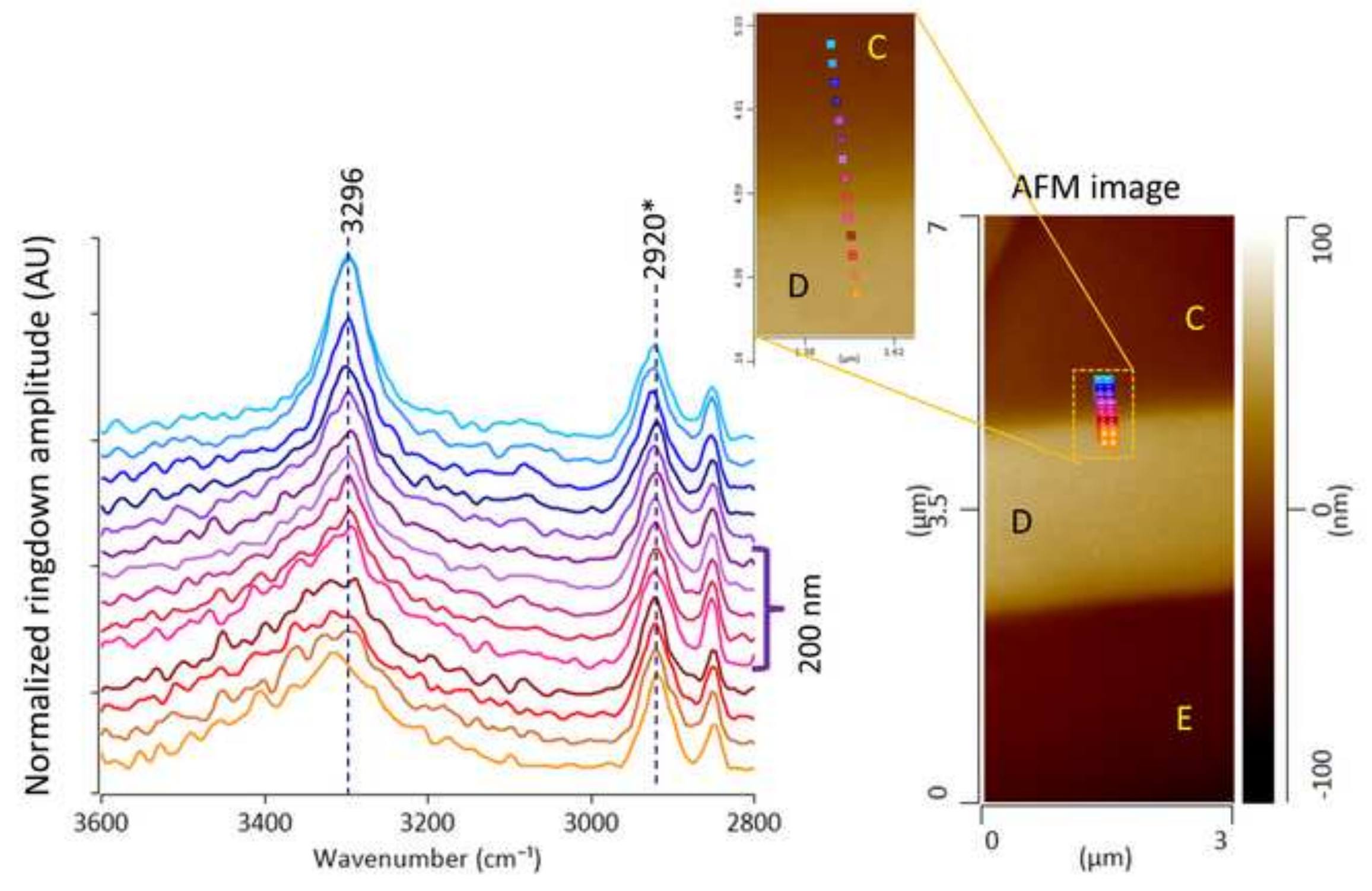

